\title{
Stellungnahme zu den Leserbriefen von Jens Alm, Hamburg, und Karsten Hartmann, Freiburg
}

Um allen Missverständnissen vorzubeugen möchten wir den folgenden Ausführungen ausdrücklich vorausschicken, dass wir eine Methodenvielfalt befürworten, in der die endovenöse Hitzeablation auch in unseren Augen einen großen Stellenwert hat. Jedes der phlebologischen Verfahren - Sklerosierung, Hitzeablation, Operation- ist aber mit seinen spezifischen Vor- und Nachteilen behaftet, die es im Sinne des Patienten abzuwägen gilt. Neben den unbestreitbaren Vorteilen der endovenösen Lasertherapie hat sich aber für diese Therapieoption der Nachteil vergleichsweise hoher Leistenrezidivraten gezeigt. Die sich daraus ergebenden Konsequenzen müssen diskutiert werden und wir freuen uns, dass mit Karsten Hartmann und Jens Alm zwei der namhaftesten Vertreter der endovenösen Therapie mit Leserbriefen Stellung bezogen haben.

In ihren Entgegnungen vertreten die beiden Autoren uns gegenüber weitgehend konträre Positionen. Konsens besteht allerdings in einem wichtigen Punkt: Alm und Hartmann sind wie wir der Ansicht, dass nicht nur bei der Operation, sondern auch bei der endovenösen Therapie kein Saphenastumpf zurückgelassen werden sollte. Wir unterstützen ausdrücklich deren Bestrebungen, durch eine „endovenöse Crossektomie“ die Qualität der Hitzeablationsverfahren zu verbessern und freuen uns, dass die lange Zeit ignorierten Erkenntnisse aus der operativen
Rezidivforschung [3] nun auch in die endovenöse Therapie Einzug halten.

\section{Zum Leserbrief von Jens Alm}

Jens Alm hat sehr frühzeitig die Problematik des „zurückgelassenen Saphenastumpfes" erkannt und spezielle Techniken entwickelt, um endovenöse Stumpfrezidive zu vermeiden.

Entgegen dem geltenden Mainstream predigt Alm seit Jahren von der Notwendigkeit einer Mitbehandlung der V. saphena accessoria anterior, um eine Situation ähnlich der operativen Crossektomie zu erreichen. Bislang ohne großes Echo.

Es wäre sehr wünschenswert, seine besondere Expertise in einer unabhängigen Multizenterstudie wiederzufinden, mit der sowohl die Wirksamkeit seiner ausgefeilten Technik, als auch deren Nachvollziehbarkeit durch andere Anwender wissenschaftlich transparent gemacht werden könnte. Solange derartige Studien aber fehlen gilt nach wie vor der gegenwärtige Erkenntnisstand, nach dem die endovenöse Lasertherapie im Vergleich zur Operation deutlich höhere Leistenrezidivraten aufweist.

Im Hinblick auf eine möglichst ambulante Leistungserbringung sind wir mit Jens Alm einer Meinung. Wenn immer möglich, sollte ambulant behandelt werden. Richtig ist auch die Aussage, die Krankenkassen könnten viel Geld sparen, wenn alle Operationen ambulant durchgeführt würden. Schließlich ist die ambulante Varizenoperation eine extrem schlecht vergütete Leistung. Für die Crossektomie und StrippingOperation (einschließlich Exhairese aller Konvolute sowie Narkose, incl. Materialzuschlag) erhalten wir (It. unserem Controlling) lediglich 668,05€. Dies ist weniger als ein Drittel im Vergleich zur stationären Operation, die uns mit knapp über $2.000 €$ vergütet wird.

Die ambulante Operation ist jedoch nicht für alle Patientengruppen geeignet. In unserer täglichen Praxis sehen wir eine große Zahl von älteren Menschen mit schlimmen Beinen oder Patienten mit besonders komplexer Varikosis, bzw. signifikanter Komorbidität. Diese Patienten werden zum Teil von niedergelassenen Phlebologen eingewiesen, die selber ambulant therapieren.

Die in diesem Zusammenhang von Jens Alm vorgeschlagene Schließung aller stationären Einrichtungen würde unsere Behandlungsmöglichkeiten deutlich einschränken und gerade diejenigen Patienten treffen, die an ihrer Varikosis am stärksten leiden. In diesem Zusammenhang darf nicht unerwähnt bleiben, dass der Zugang zur stationären Behandlung einer strengen Regulierung unterliegt, nach der ausschließlich Patienten aufgenommen werden können, 
bei denen Ausnahmetatbestände vorliegen. Wenn diese nicht nachgewiesen werden können, wird dem Krankenhaus lediglich die ambulante Behandlung erstattet. In Einzelfällen mag es möglich sein, diese strengen Regeln zu umgehen. In Bochum werden jedoch so gut wie alle stationär behandelten Fälle von den Medizinischen Diensten der Krankenkassen überprüft.

\section{Zum Leserbrief von Karsten Hartmann}

Die von Karsten Hartmann angeführte Polarisierung sehen wir eher positiv. Schließlich kann eine wissenschaftliche Diskussion nur dann aufkommen, wenn unterschiedliche Pole existieren. Mainstream hilft uns nicht weiter. Allerdings haben wir in unserer Publikation - entgegen den Ausführungen von Karsten Hartmann - nicht einfach nur Behauptungen aufgestellt, sondern den aktuellen Stand aus den verfügbaren Vergleichsstudien (RCT) wiedergegeben. Karsten Hartmann hat selber an der umfangreichsten RCT mitgewirkt [1]. In dieser Studie waren die duplexsonographisch detektierbaren Leistenrezidive nach Lasertherapie viermal häufiger als nach der Operation. Genau diese Arbeit bildete die Grundlage für unsere Berechnungen.

Darüber hinaus haben wir in unserer Publikation dargelegt, in welchem Kontext die im Vergleich hohen Leistenrezidivraten nach Lasertherapie zu sehen sind. Auch diese Betrachtungen belegten wir mit Fakten. Hierzu diente uns im Wesentlichen die „Deutsche Leistenrezidivstudie“ [2], die weltweit umfangreichste Multizenterstudie mit histopathologischer Aufarbeitung von Stumpfrezidiven, zu der Michael Hartmann einen wesentlichen Beitrag leistete.

Karsten Hartmann vertritt in seinen Ausführungen die Ansicht, dass mit der sogenannten „endovenösen Crossektomie“ das Gleiche geleistet werden könnte wie mit der operativen Crossektomie, nur eben schonender und kostengünstiger. Dies ist allerdings eine Hypothese, die lediglich auf der persönlichen Einschätzung des Autors basiert. Für einen wissenschaftlichen Beweis der Gleichwertigkeit des Lasers wären aber unabhängige Vergleichsstudien mit Evaluation der Langzeitergebnisse erforder- lich. Solange es einen solchen Beweis nicht gibt, dürfen wir unseren Patienten gegenüber nicht von einer Gleichwertigkeit der Verfahren sprechen. Ansonsten würden wir den Fehler wiederholen, der schon bei der Markteinführung des Lasers der ersten Generation begangen wurde. Damals wurde der Laser den Patienten als überlegene Therapieoption angepriesen. Heute wissen wir, dass dies nicht richtig war. Der Laser der ersten Generation gilt sogar als Flop.

Wir sollten aus diesem Fehler lernen und neue Verfahren vor ihrer Markteinführung evaluieren, damit wir unsere Patienten auf der Basis solider Daten informieren können.

Ein wesentlicher Teil der Ausführungen von Karsten Hartmann betrifft die Kosten der endovenös thermischen Therapie im Vergleich zum „chirurgischen Gegenpart“. Diese Betrachtungen waren eigentlich nicht Bestandteil unserer Publikation, in der es lediglich um die Folgekosten von Leistenrezidiven ging. Für die Bewertung dieser Folgekosten griffen wir, wie in der Publikation beschrieben, auf die beiden deutschen Vergleichsstudien zurück. Schließlich wollten wir die deutsche Situation mit ihren Folgekosten abbilden.

Zu den Ausführungen von Karsten Hartmann bezüglich des Kostenvergleiches zwischen der operativen und der endovenösen Therapie sind wir grundsätzlich anderer Meinung.

Der Autor vergleicht die ambulante endovenöse Therapie (ca. 1500€) mit einer stationären operativen Therapie (ca. $2000 €$ ). Die stationäre Therapie umfasst aber ein anderes Krankengut als die ambulante, nämlich ausschließlich Patienten mit besonders komplexer Erkrankung oder erheblicher Komorbidität. Wie bereits in der Stellungnahme zum Leserbrief von Jens Alm ausgeführt, unterliegt der Zugang zur stationären Therapie einer strengen Regulierung. Nach unserer Einschätzung ist daher ein Vergleich zwischen ambulanter Hitzeablation und stationärer Operation nicht statthaft.

Stellt man aber einen Vergleich der ambulanten endovenösen Therapie mit der ambulanten Varizenoperation an, so ist der von Karsten Hartmann genannte Kostenvorteil endovenöser Verfahren nicht er- kennbar. In Relation zu den genannten $1.500 €$ für einen endovenösen Eingriff sind die Kosten des ambulanten Operierens nach $\S 115$ b SGB V mit 668,05€ deutlich niedriger.

\section{Literatur}

[1] Flessenkämper I, Hartmann M, Hartmann K et al. Endovenous laser ablation with and without high ligation compared to high ligation and stripping for treatment of great saphenous varicose veins: Results of a multicentre randomised controlled trial with up to 6 years follow-up. Phlebology 2016; 31 (1): 23-33

[2] Mumme A, Burger P, Hummel T et al. High ligation of the saphenofemoral junction is necessary! Results of the German Groin Recurrence Study. Phlebologie 2009; 38: 99-102

[3] Mumme A, Burger P, Hummel T et al. Der lang belassene Saphenastumpf - Implikationen für die endovenöse Therapie der Varikosis. Phlebologie 2007; 36: 256-259

\section{Interessenkonflikt}

Der Autor gibt an, dass kein Interessenkonflikt besteht.

\section{Autorinnen/Autoren}

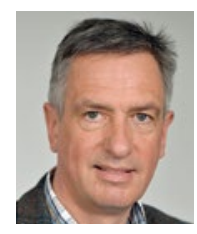

\section{Prof. Dr. med.}

Achim Mumme

Klinik für Gefäßchirurgie Katholisches Klinikum der Ruhr-Universität Bochum, Standort St. Josef-Hospital Gudrunstr. 56 44791 Bochum E-Mail: achim.mumme@ruhruni-bochum.de

Bibliografie

DOI https://doi.org/10.1055/a-0890-9028

Phlebologie 2019; 48: 195-196

(c) Georg Thieme Verlag KG Stuttgart · New York ISSN 0939-978X

\section{Publikationshinweis}

Leserbriefe stellen nicht unbedingt die Meinung von Herausgebern oder Verlag dar. Herausgeber und Verlag behalten sich vor, Leserbriefe nicht, gekürzt oder in Auszügen zu veröffentlichen. 